\title{
Research and Application of Enterprise Knowledge Management System Based on Ontology
}

\author{
Tiedong Chen', Ziyu Liu ${ }^{2}$ and Lei Huang ${ }^{1}$ \\ ${ }^{1}$ College of Economics and Management, Beijing Jiaotong University, Beijing 100044, P.R. \\ China Chentiedong815@sina.com \\ ${ }^{2}$ College of Information Science \& Engineering, Hebei University of Science \& \\ Technology, Shijiazhuaug 050018, P.R. China
}

\begin{abstract}
With the coming of knowledge economy, the pattern of enterprise management is going through a great change. Nowadays, in an enterprise, knowledge management has become a key technology to increase intelligence of technique, competition, business and strategy. The key factors for the enterprise to survive the competitive environment lie in how to extract the needed knowledge from numerous of external and internal information, and how to manage the acquiring, producing and spreading of knowledge and the ability to upgrading of the enterprises based on knowledge. This paper proposes an ontology-based knowledge management framework. Then an application case is presented, which is an examples of knowledge management system based on ontology for a logistics enterprise.
\end{abstract}

Keywords: Knowledge management, Ontology, Knowledge acquisition and collation, Knowledge storage, Knowledge reuse

\section{INTRODUCTION}

Facing economic globalization and fierce market competition, knowledge-intensive enterprises have paid attention to which how to effectively reuse relevant knowledge that are included in the enterprise and outside information system to improve operating efficiency and market competitiveness. More and more enterprises hope that their core competitiveness should be founded on the basis of knowledge innovation, so they want to adopt a knowledge management strategy to achieve this purpose [1]. Under such circumstances, knowledge management technology emerged. Knowledge management was raised earliest by the United Nations International Labor Organization in 1986. In the enterprise context, we adopt the definition of knowledge management as a "concerted effort to capture, organize and share what they know" [2].

Under such circumstances, how to effective retrieve information has become an important research topic [3]. Ontology can solve this problem. Ontology can determine the precise meaning of concept through the strict definition for concept and the relationship between these concepts and these concepts can express the knowledge that can be common recognized and shared. The efficiency of knowledge search, knowledge accumulation and knowledge sharing will be greatly enhanced under the

Please use the following format when citing this chapter:

Chen, T., I.iu, 7.., Huang, I.., 2007, in IFIP International Federation for Information Processing, Volume 254, Research and Practical Issues of Enterprise Information Systems II Volume I, eds. I. Xu, Tjoa $\Lambda$.. Chaudhry S. (Boston: Springer), pp. 747-751. 
support of such a series of concepts, so that the true meaning of knowledge reuse and knowledge sharing become possible. In this paper an application case is presented, which is an examples of knowledge management system based on ontology for a logistics enterprise.

\section{ONTOLOGY}

Ontology that originates from philosophy is widespread paid attention to among the field of information science in recent years [4-5], and its importance has been demonstrated in many ways and has been widely recognized [4][6-7]. Ontology can be regarded as a vocabulary of terms and relationships between those terms in a given domain. Ontology facilitates capture and construction of domain knowledge and enables representation of skeletal knowledge to facilitate integration of knowledge bases irrespective of the heterogeneity of knowledge sources. There are a number of definitions about the ambiguous term "ontology", and Gruber's definition is at large accepted: ontology is an explicit specification of a conceptualization.

\section{PROPOSED SOLUTION}

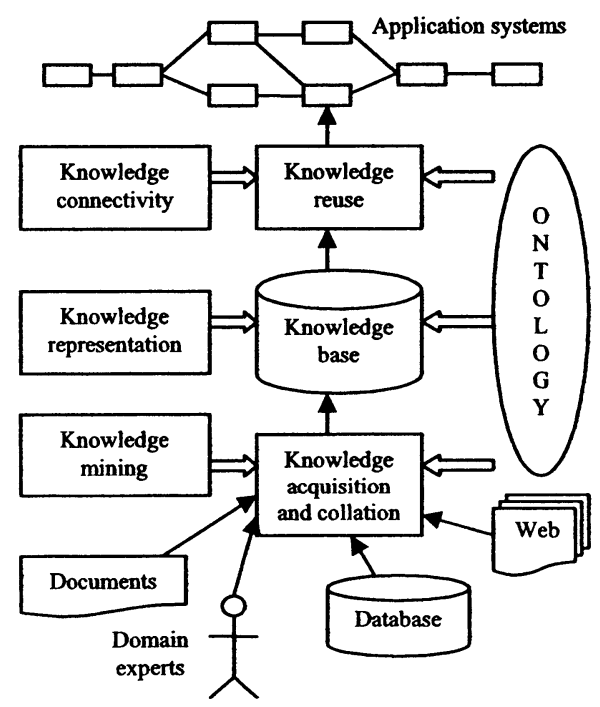

Figure 1. Open Knowledge Management Architecture Based on Ontology

In our ontology-based enterprise knowledge management frame, the knowledge management process is concisely divided into three phases: knowledge acquisition 
and collation, knowledge storage and knowledge reuse. It uses knowledge connectivity and transformation mechanisms to drive the process of acquisition and reuse. The system architecture is shown in figure 1 , and in figure 1 the fine black lines indicate knowledge flow.

\subsection{Knowledge Acquisition and Collation}

Knowledge acquisition and collation refers to adding meta-information to information. It is a transformation process from structured, semi-structured and unstructured information to the structured information. According to the source of information, there are four categories: (1) Background information of individuals and groups. (2) Various homogeneous or heterogeneous databases. (3) Various documentations. (4) Knowledge over the web including information that is originated from data mining [8].

Knowledge acquisition is the foundation and main technology of knowledge management systems, and it includes knowledge extraction and knowledge acquisition form knowledge base. Knowledge extraction is knowledge's abstract process in which knowledge is extracted from the raw data and information according to concepts that are defined by ontology. In traditional knowledge engineering there are a number of mature knowledge extraction methods and tools, such as Text Miner of IBM, OntoEdit of OntoPrise and Protégé of StanFord [9]. The original data and information may not be electronic, and electronic information may be structured, semi-structured or unstructured information. Non-electronic information can be transformed into structured information by ontology developing tools or OCR tools.

\subsection{Knowledge Storage}

Knowledge storage can store the knowledge that has been transformed in knowledge base. The knowledge that has been transformed is divided into two parts: one is structured meta-knowledge, the other is semi-structured or unstructured information.

\subsection{Knowledge Reuse}

Knowledge reuse is the process of using knowledge in application systems, and the reuse of knowledge is achieved by the link between people and knowledge. There are four mainly link: (1) Linking people to knowledge. You can find relevant content at different levels by using visual query and retrieval tools, which is acquiring knowledge through pull. (2) Linking people to people. Knowledge workers can find relevant experts and result to or discuss with them through knowledge management system. (3) Linking knowledge to people. Knowledge management system can push the relevant knowledge to people according to personal preference or immediate needs. (4) Linking knowledge to knowledge. It achieves hyperlink between knowledge and knowledge. 


\section{CASE STUDY}

On the basis of the frame that we proposed, we developed a knowledge management system of three-layer structure for a logistics enterprise. As shown in Figure 2. At the client user agent send the request to intermediate layer through SOAP protocol. The middle layer search the required knowledge from ontology knowledge base according to the user's request and return them to the users. The middle layer also can automatically acquire knowledge from the outside and store them in the knowledge base. The database layer is responsible for the storage and sharing of knowledge. The specialized knowledge engineers are responsible for the work of knowledge maintenance.

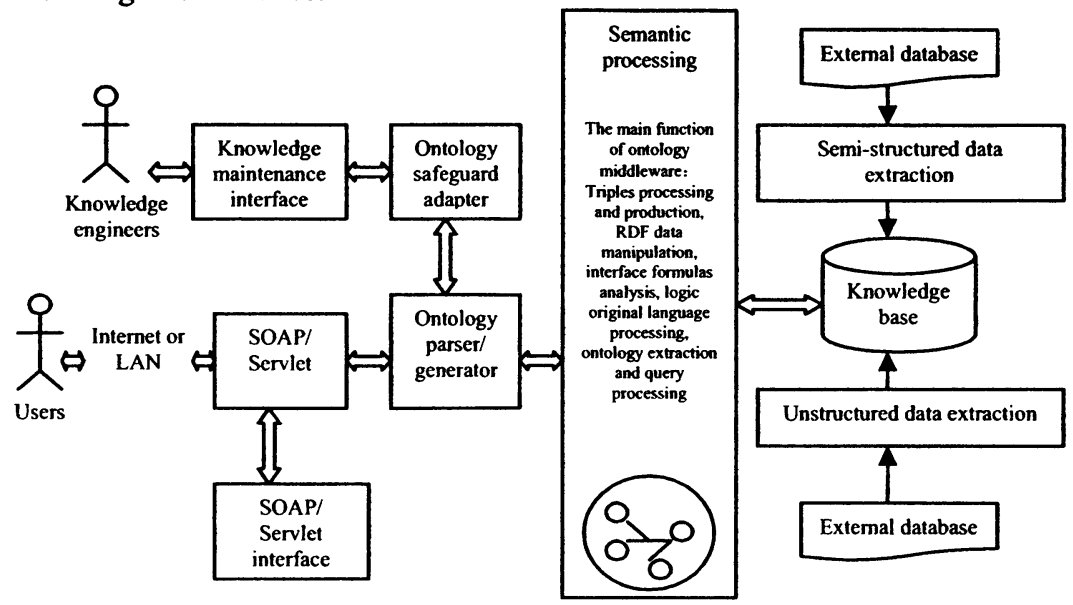

Figure 2. Knowledge Management System Based on Ontology

The function of the main module:

(1) SOAP / Servlet: Finishing the client agency, automatically adapting the changes of the client and finishing interaction with the server's servlet are its main function [2].

(2) SOAP / Servlet interface: These interfaces are application program interfaces that are defined to meet the requirements of knowledge management system, such as query interface, visit interface etc. It can recall the corresponding interface processing according to customer request.

(3) Ontology parser/generator: It is responsible for parsing or generating ontology information.

(4) Ontology middleware: It is the core of knowledge management system. It is responsible for extracting knowledge information from knowledge.warehouse and achieving the knowledge's sharing and using. It has the function of querying and reasoning, and it can directly connect with the database system.

(5) Knowledge warehouse: It is responsible for storing knowledge information and achieving the storage and sharing of knowledge. 
Research and Application of Enterprise Knowledge Management System Based on

Ontology 751

(6) Unstructured/semi-structured data extraction module: It is responsible for extracting knowledge information from external data sources and storing them in knowledge base.

\section{CONCLUSIONS}

At present, the internet is moving forward the direction of the semantic web, and knowledge management is increasingly becoming the core of enterprise management. Ontology can solve a series of issues of knowledge management. Because the current knowledge management systems have a common limitation: they are insufficient in dynamic interaction with users, which is denoted by the static usage of knowledge and the closed maintenance of system. According to this, we construct an ontologybased open knowledge management system. On the basis of these, we developed a knowledge management system based on ontology for a logistics enterprise. The practical application shows that system has achieved expected results for supporting compatibility matching, meaning understanding, knowledge networking and establishing and maintaining ontology of knowledge workers etc.

\section{REFERENCES}

1. F. Li, J. Gao, L. Zhong, and M. Zhou, OKMF: an ontology-based knowledge management system framework, Journal of computer-aided design \& computer graphics. Volume 15, Number 12, pp.1538-1543, (2003).

2. A. Stuart, Knowledge Management Uneasy Pieces, C10 Magazine site on the World Wide Web, Part 2 (1996). http ://www. cio. com/CIO/060196_uneasy_ 1 html (Accessed December 7, 1996)

3. B. Shen, Research on knowledge management system of enterprise based ontology, National Business: Research on Economic Theory. Number 2, pp.70-72, (2006).

4. N. Guarino, Formal ontology and information systems, in Proc of the lst Int'l Conf on Formal Ontology in Information Systems, Trento (IOS Press: Italy, 1998), pp.3-15.

5. M. Uschold and M. Gruninger, Ontologies: Principles, methods, and applications, Knowledge Engineering Review. Volume 11, Number 2, pp.93-155, (1996).

6. T.B. Lee, J. Hendler, and O. Lassila, The semantic Web, Scientific American. Volume 284, Number 5, pp.34-43, (2001).

7. T.B. Lee, Semantic Web road map (1998). http://www.w3.org/DesignIssues/Semantic.html

8. B. Liu, Study on key technologies of ontology based knowledge management, JCSSTI. Volume 24, Number 1, pp.75-81, (2005)

9. X. Huang, $X . X u$, and $G$. Xu, Research of knowledge management system based ontology, Science technology and engineering. Volume 5, Number 6, pp.351-356, (2005). 